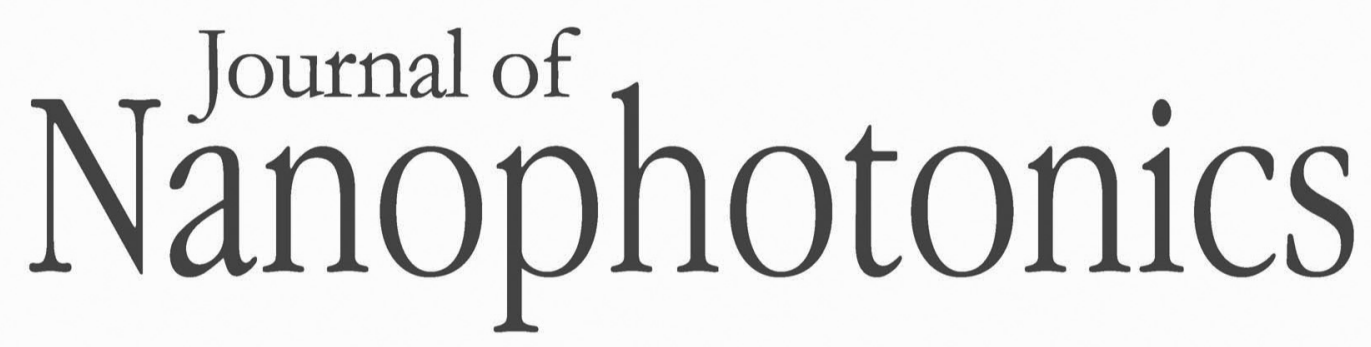

SPIEDigitalLibrary.org/jnp

\title{
Label-free photonic biosensors fabricated with low-loss hydrogenated amorphous silicon resonators
}

Timo Lipka

Lennart Wahn

Hoc Khiem Trieu

Lutz Hilterhaus

Jörg Müller 


\title{
Label-free photonic biosensors fabricated with low-loss hydrogenated amorphous silicon resonators
}

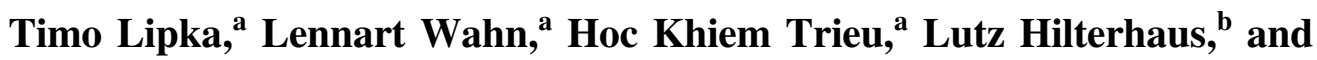 \\ Jörg Müller ${ }^{a}$ \\ ${ }^{a}$ Hamburg University of Technology, Institute of Micro System Technology, \\ Eissendorferstrasse 42, 21073 Hamburg, Germany \\ timo.lipka@tuhh.de \\ ${ }^{b}$ Hamburg University of Technology, Institute of Technical Biocatalysis, \\ Denickestrasse 15, 21073 Hamburg, Germany
}

\begin{abstract}
The precise detection of chemicals and biomolecules is of great interest in the areas of biotechnology and medical diagnostics. Thus, there is a need for highly sensitive, small area, and low-cost sensors. We fabricated and optically characterized hydrogenated amorphous silicon photonic resonators for label-free lab-on-chip biosensors. The sensing was performed with small-footprint microdisk and microring resonators that detect a refractive-index change via the evanescent electric field. Homogeneous sensing with $\mathrm{NaCl}$ and surface-sensing experiments with immobilized bovine serum albumin (BSA) were carried out. A sensitivity as high as $460 \mathrm{~nm} / \mathrm{RIU}$ was measured for $\mathrm{NaCl}$ dissolved in deionized water for the disk, whereas about $50 \mathrm{~nm} / \mathrm{RIU}$ was determined for the ring resonator. The intrinsic limits of detection were calculated to be $3.3 \times 10^{-4}$ and $3.2 \times 10^{-3}$ at $1550-\mathrm{nm}$ wavelength. We measured the binding of BSA to functionalized ring resonators and found that molecular masses can be detected down to the clinically relevant femtogram regime. The detection and quantification of related analytes with hydrogenated amorphous silicon photonic sensors can be used in medical healthcare diagnostics like point-of-care-testing and biotechnological screening. (C) The Authors. Published by SPIE under a Creative Commons Attribution 3.0 Unported License. Distribution or reproduction of this work in whole or in part requires full attribution of the original publication, including its DOI. [DOI: 10.1117/1.JNP.7.073793]
\end{abstract}

Keywords: amorphous silicon; a-Si:H; photonics; microring; microdisk; resonator; label-free biosensor; lab-on-chip; point-of-care diagnostics.

Paper 13050SSP received Jun. 27, 2013; revised manuscript received Oct. 5, 2013; accepted for publication Oct. 7, 2013; published online Nov. 5, 2013.

\section{Introduction}

Photonic biosensors, which are fabricated with high refractive index photonic resonators, have good prospects in the area of optical evanescent field sensing applications. In particular, silicon is a promising platform to fabricate photonic lab-on-chip sensors for chemical, medical, and biosensing. The compatibility with mature CMOS technology provides the basis for mass fabrication at low cost and the possibility to fabricate large sensor arrays on a common substrate with ultra-compact sized resonators $(\leq 5 \mu \mathrm{m})$ that additionaly offer high sensitivities and low detection limits. ${ }^{1,2}$

Instead of using crystalline silicon-on-insulator (SOI) wafers as photonic substrates, low-loss hydrogenated amorphous silicon $(\mathrm{a}-\mathrm{Si}: \mathrm{H})$ that is deposited by plasma-enhanced chemical vapor deposition (PECVD) is alternatively employed in this work because it provides a high refractive index $(n \approx 3.5)$ and low-loss material for near-infrared photonics, both comparable to crystalline silicon. ${ }^{3}$ However, a-Si:H offers a more flexible fabrication process since it can be deposited at relatively low temperatures $\left(\leq 300^{\circ} \mathrm{C}\right)$ and therefore allows using glass or plastic materials as substrates. ${ }^{4,5}$ This facilitates a low-cost fabrication and offers more processing options, e.g., for the combination with microfluidic channels using split-and-recombine micromixers or for the arrangement of the sensing resonators that can be principally fabricated in a vertically stacked configuration. ${ }^{6,7}$ 
In this paper, we present a versatile and CMOS-compatible photonic lab-on-chip platform, which utilizes high-quality a-Si:H photonic resonators as the biosensing elements. Therefore, photonic microdisk and ring resonators were fabricated. The photonic systems were patterned with electron beam lithography and structured with inductively coupled plasma (ICP) etching, resulting in $480 \times 200 \mathrm{~nm}$ photonic wire waveguides and disk and ring resonators with 10 and $20 \mu \mathrm{m}$ radius, respectively. The resonators operate near critical coupling and show extinction ratios up to $25 \mathrm{~dB}$ with an aqueous top cladding of deionized (DI) water, which is beneficial in order to minimize amplitude and phase noise limiting the sensitivity and detection limit. The a-Si:H resonators exhibit loaded $Q$-factors of $\approx 10^{4}$ for both resonator types and both polarizations.

From homogeneous sensing experiments with different sodium chloride $(\mathrm{NaCl})$ concentrations, a disk resonator sensitivity of $460 \mathrm{~nm} / \mathrm{RIU}$ was determined, whereas the $10-\mu \mathrm{m}$ ring resonators exibited $50 \mathrm{~nm} /$ RIU. To prove the feasibility to detect specific biomolecules, e.g., enzymes binding to the resonator cavity, the a-Si:H sensor surface of a $20-\mu \mathrm{m}$ ring resonator was modified by a silanization step with alkoxysilane molecules. Glutaraldehyde was then utilizied as an amine-reactive homobifunctional-crosslink in order to allow the binding of the targeted bovine serum albumin (BSA) molecules. After exposing the functionalized sensor surface for $1 \mathrm{~h}$ in a BSA solution, a wavelength shift of $1.25 \mathrm{~nm}$ was measured from which a limit of detection in terms of surface coverage of $8.5 \mathrm{pg} / \mathrm{mm}^{2}$ is determined.

\section{On-Chip Photonic Sensing Platform}

\subsection{Hydrogenated Amorphous Silicon Photonic Sensing Platform}

Different kinds of transparent optical materials like nitrides, polymers, glasses, and others have been already used to fabricate photonic resonators for lab-on-chip sensing applications. ${ }^{8,9}$ However, there are essential advantages of employing a high refractive index material like silicon, which are of economic and physical nature.

The main economic reason is the compatibility of advanced CMOS-technology with welldeveloped infrastructure and long-time process experience, which will allow sharing existing facilities with electronics, or to combine electronic and photonic systems on the same substrate. Furthermore, the high index contrast (HIC) between waveguide core and cladding materials (e.g., $\mathrm{Si}$ and $\mathrm{SiO}_{2}$ with $\Delta n \geq 2$ ) permits a high integration density and thus photonic systems with low footprint so that hundreds of sensor arrays with multiple channels can be cost effectively fabricated on a single wafer. This makes the silicon platform interesting for applications like point-of-care diagnostics. However, the HIC is just as much beneficial in terms of the sensor sensitivity. The strong mode confinement in single-mode photonic wire waveguides with typical dimensions of $500 \times 200 \mathrm{~nm}$ allows for small bending radii $R \leq 5 \mu \mathrm{m}$ with negligible losses of $\approx 0.1 \mathrm{~dB}$ per revolution. As a result of that, a large free spectral range of $\approx 18 \mathrm{~nm}$ can be achieved with such ring resonators, which facilitates a distinct detection of large analyte changes and favors the interrogation of several resonator-based sensors with one readout waveguide.

Addditionally, the tight light guidance implies that there is a strong electric field discontinuity at the low and high refractive index layer interfaces and thus the evanescent field can effectively interact with the analyte medium which covers the photonic sensor. The sensitivity enhancement can be expressed in terms of the evanescent field strength according to

$$
\left|\frac{E_{\text {clad }}}{E_{\text {core }}}\right|=\left(\frac{n_{\text {core }}}{n_{\text {clad }}}\right)^{2},
$$

with $E_{\text {clad }}$ and $n_{\text {clad }}$ as the electric field strength and the refractive index in the low index region, and $E_{\text {core }}$ and $n_{\text {core }}$ the corresponding values of the high index region. ${ }^{10}$ The typical photonic wires guide two fundamental modes, the quasi transverse electric (qTE) and the quasi transverse magnetic modes (qTM), which are, due to convention, distinguished by the electric field orientation predominantly parallel and perpendicular to the substrate. The modes of a $480 \times 200 \mathrm{~nm}$ dimensioned photonic wire were simulated at $1550-\mathrm{nm}$ wavelength by using a finite element method (FEM) mode solver and the results are presented in Figs. 1(a) and 1(b). For the 
Lipka et al.: Label-free photonic biosensors fabricated with low-loss hydrogenated...
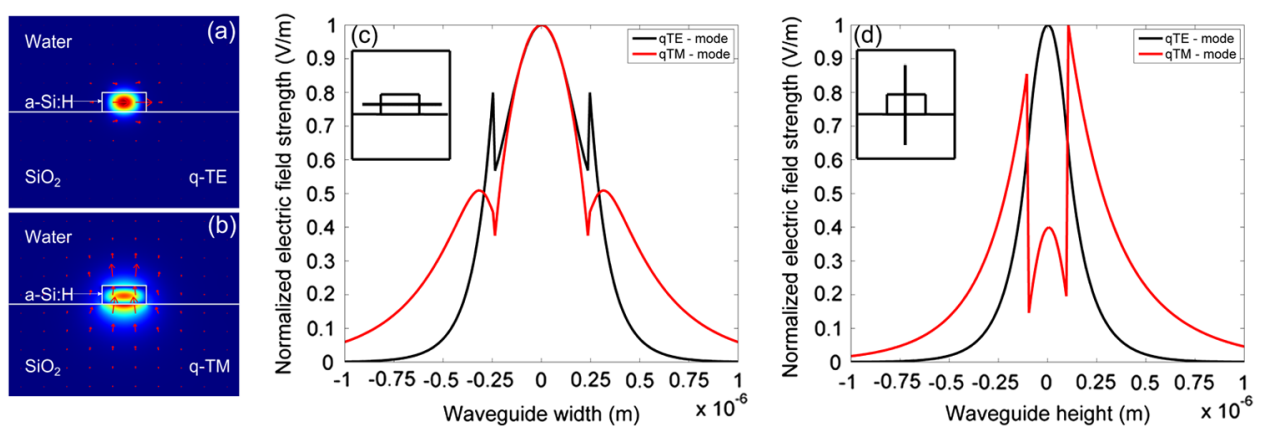

Fig. 1 Results of finite element method (FEM) simulations: Fundamental qTE (a) and qTM (b) guided mode intensity plots for an a-Si:H photonic waveguide with $480 \times 200 \mathrm{~nm}$ dimensions and water cladding with electric field orientations inset as arrows. Normalized electric field strength line plots of the fundamental modes for the width (c) and the height (d) of the photonic wires.

Table 1 Effective mode indices and confinement factors for both fundamental modes of a $480 \times$ $200 \mathrm{~nm}$ dimensioned a-Si:H photonic wire with oxide under and aqueous top cladding.

\begin{tabular}{lcc}
\hline \hline Mode & Effective mode index $\left(N_{\text {eff }}\right)$ & Confinement factor $(\Gamma)$ \\
\hline qTE & 2.308 & 0.749 \\
qTM & 1.566 & 0.245 \\
\hline \hline
\end{tabular}

simulations, the bulk refractive indices of the materials were implemented as follows: $n=3.478$ for the amorphous silicon core, $n=1.444$ for the oxide under cladding, and $n=1.31$ for the water top cladding. The effective mode indices and the mode confinement factors, which are a measure of the light guided inside the core, were determined as well and are summarized in Table 1 . The intensity plots indicate that for the given dimensions, the qTE mode is stronger confined compared to the qTM mode, which decreases the interaction of this mode with the sensing medium. The normalized electric field profiles of both modes, which show the evanescent field distribution for a line plot across the photonic waveguides width and height, are shown in Figs. 1(c) and 1(d).

\subsection{Sensing Principle and Figures of Merit}

Photonic evanescent field refractive index sensors can be categorized due to different sensing principles as shown in Fig. $2 .{ }^{11}$ Surface sensing is a method where selective receptor molecules
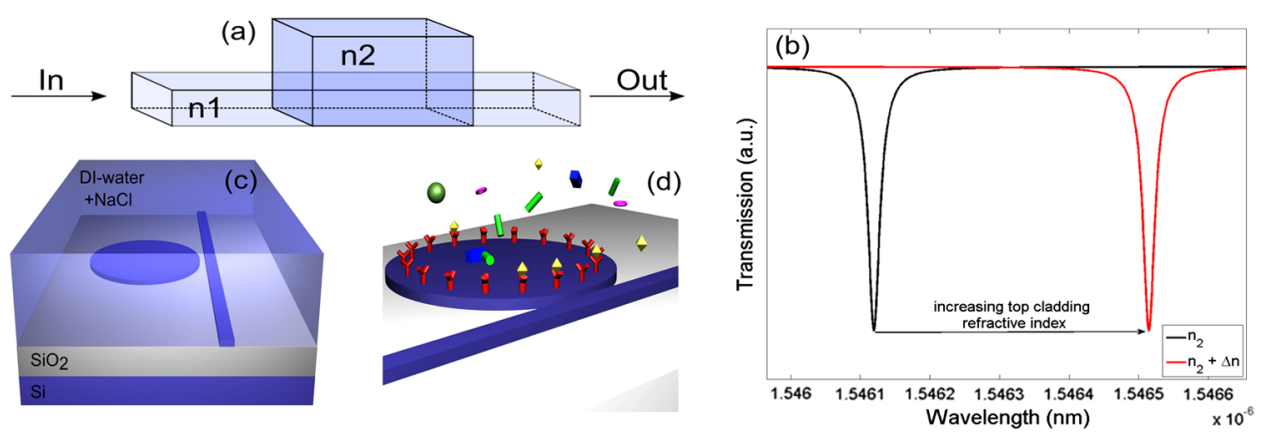

Fig. 2 (a) and (b) Illustration of the sensing principle by a change of cladding refractive index which results in a wavelength shift. Scheme of (c) homogeneous sensing method and (d) of surface sensing method. 
are immobilized on a functionalized photonic device surface so that only target molecules will selectively stick to the receptors. This way the propagation of the guided mode ( $\left.N_{\text {eff }}\right)$ is slightly altered and hence, the response of the photonic sensor is a measure of the adsorpted molecule amount. The surface sensing technique is very promising for lab-on-chip or point-of-care biosensing applications. Compared to that, homogeneous sensing might be employed to measure gases and chemicals. In homogeneous sensing, the photonic sensor is covered with a cladding medium, e.g., water or air, and a change of a specific concentration in the medium (e.g., $\mathrm{NaCl}$ ) is detected by the refractive index difference $\Delta n$ of the analyte. For both the methods, the interrogation of the sensor can be performed with spectral transmission measurements and the signal can be evaluated either by a resonance peak shift or by an increase in absorption, for example, induced by gases in the mid-infrared. In this work, homogeneous sensing was employed in order to detect the refractive index change by measuring the resonance wavelength shift due to different $\mathrm{NaCl}$ concentrations. The intrinsic sensitivity $\left(S_{\mathrm{RIU}}\right)$ of the resonator-based refractive index sensor can be defined as

$$
S_{\mathrm{RIU}}=\frac{\Delta \lambda_{\text {res }}}{\Delta n_{\text {fluid }}},
$$

with $\Delta \lambda_{\text {res }}$ as peak shift of a resonant mode that is related to a difference in the fluids refractive index $\Delta n_{\text {fluid }}$. The minimum detectable change $\Delta n_{\min }$ for a given wavelength $\lambda$, the limit of detection (LoD), is calculated as follows:

$$
\mathrm{LoD}=\Delta n_{\min }=\frac{\lambda}{Q S_{\mathrm{RIU}}},
$$

with $Q$ as quality factor of the resonance peak, which corresponds to the photon lifetime inside the resonator and therefore determines the effective interaction length with the analyte, and that is defined as ratio of the resonant peak position and the full width half maximum or 3-dB bandwidth $\Delta \lambda_{3 \mathrm{~dB}}$ :

$$
Q_{\text {loaded }}=\frac{\lambda_{\text {res }}}{\Delta \lambda_{3 \mathrm{~dB}}}
$$

From the set of Eqs. (1)-(4), it is evident that a high sensitivity is achieved when there is a strong modal overlap of the guided mode with the sensing medium and that additionally a high $Q$ resonator will enhance the LoD. However, a high $Q$ factor does not necessarily improve the sensitivity, especially for water-based solutions, because water adds substantial propagation loss $\left(\approx 10 \mathrm{~cm}^{-1}\right.$ at $\left.1550 \mathrm{~nm}\right)$, and a high quality factor often results from a stronger mode confinement in the resonator core and hence less light can effectively interact with the sensing medium. ${ }^{12,13}$

Based on these considerations, the homogeneous sensing experiments were carried out with microdisk resonators which exhibit moderate $Q$-factors of $10^{4}$ although we have already fabricated critically coupled disk resonators with higher intrinsic $Q$ of $\geq 10^{5}$ for qTE and $\geq 0.9 \times 10^{6}$ for qTM with amorphous silicon. ${ }^{14}$ In order to achieve a strong interaction of the evanescent field with the sensing medium, the following disk resonator experiments were conducted for the qTMmode. The disk radius was chosen to be $R=10 \mu \mathrm{m}$ so that a sufficiently large free spectral range $(\mathrm{FSR}=10 \mathrm{~nm})$ for resonances of the same modal order was achieved. The coupling gap between disk and waveguide was designed to be $280 \mathrm{~nm}$ for weak coupling.

\subsection{Functionalization of the Resonators}

The sensor chip was functionalized through following process steps for the detection of BSA proteins. First, the chip surface was cleaned with Piranha-etch followed by several wash steps. Then, the a-Si:H sensor surface was modified by a silanization step with alkoxysilane molecules (APTES). Glutaraldehyde was utilizied as an amine-reactive homobifunctional-crosslink in order to allow the binding of the targeted BSA molecules. The process procedure is schematically summarized in Fig. 3(a). In order to verify the binding of the BSA-proteins on a-Si:H chips, 
Lipka et al.: Label-free photonic biosensors fabricated with low-loss hydrogenated...
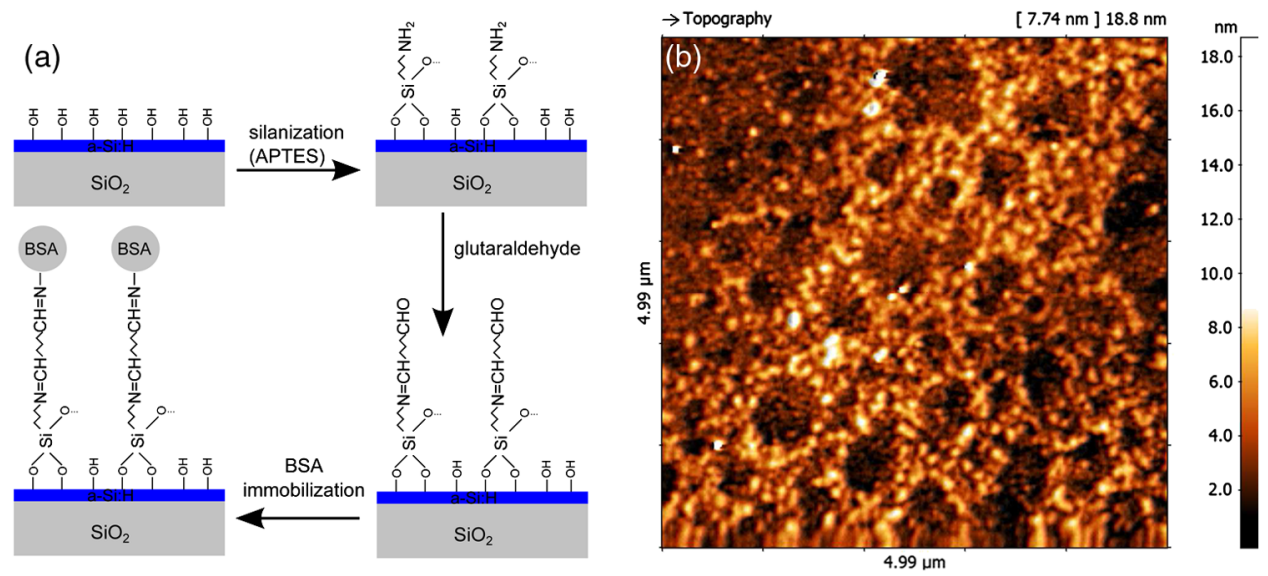

Fig. 3 (a) Functionalization procedure using silanization (APTES) and amine-reactive homobifunctional-crosslinking. (b) Atomic force microscope picture of immobilized bovine serum albumin (BSA) on a-Si:H sample.

fluorescent measurements of the supernatant were performed. The FluoroProfile Protein Quantification Kit (Sigma-Aldrich, Germany) used is based on the epicocconone. The epicocconone represents a fluorescent probe based on a polyketide skeleton isolated from the fungus Epicoccum nigrum and produces a large increase in fluorescence quantum yield upon binding to detergent-coated proteins in solution. ${ }^{15}$ The protein quantification was carried out in accordance with the supplier's protocol. An atomic force microscope (AFM) picture with a $5 \times 5 \mu \mathrm{m}$ scan area of a sample with adsorbed BSA proteins is shown in Fig. 3(b).

\section{Photonic Sensor Fabrication and Optical Characterization}

\subsection{Layer Deposition and Photonic Sensor Fabrication}

Standard crystalline silicon wafers $(10 \mathrm{~cm})$ with a $3 \mu \mathrm{m}$ thermal grown bottom oxide cladding layer were used as substrates. Thus, for this refractive index contrast and layer thickness, substrate leakage is effectively supressed $(\leq 0.01 \mathrm{~dB} / \mathrm{cm})$ for both fundamental modes (qTE and qTM). The PECVD chamber was conditioned with the optimized a-Si:H material in order to achieve stable plasma conditions prior to the actual core layer deposition. After that, the a-Si:H core layer was deposited with an argon/silane plasma at a temperature of $300^{\circ} \mathrm{C}$. The refractive index $n$ and the thickness $t$ of the a-Si:H layers were controlled with ellipsometry at $1534 \mathrm{~nm}$ resulting in highly uniform photonic substrates with $n=3.48$ and $t=200 \mathrm{~nm}^{16}$

The absorption loss of the optimized a-Si:H material has been already reported to be lower than $0.5 \mathrm{~dB} / \mathrm{cm}$ at $1550-\mathrm{nm}$ wavelength. ${ }^{3}$ The photonic wires and the disk resonators were patterned with positive photoresist and electron beam lithography at Heinrich Hertz Institute, Berlin. In order to smooth the photo resist and to minimize line-edge roughness at the photonic wire sidewalls, a short descum process with $\mathrm{O}_{2}$ plasma with $80-\mathrm{W}$ coil power was applied. The a-Si:H core layer was then structured by highly anisotropic dry etching process with a continuous flow of $\mathrm{C}_{4} \mathrm{~F}_{8}$ and $\mathrm{SF}_{6}$ in an ICP, which allows for smooth and straight sidewalls. The residual resist was removed by an oxygen plasma, and the wafer was fragmented into photonic chips, which were cleaned with wet chemicals and were left uncladded for the optical measurements.

\subsection{Optical Measurement Setup}

The microdisk and microring spectra were characterized with optical transmission measurements. Closed-loop controlled piezo-electric alignment stages and different cameras were used to maximize the coupling to the photonic chip. The photonic sensors were placed on a thermo electric controller (TEC) temperature stabilized chip mount such that a constant temperature with variations less than $0.01^{\circ} \mathrm{C}$ could be maintained during the experiments. In case of the 
disk resonators, a lensed fiber was used to launch the NIR-light (Agilent 8164B) into the cleaved facets of the supporting photonic wire waveguides. A microscope objective with high numerical aperture of NA $=0.65$ was utilized to collect the output signal and guide it to a fiber-coupled collimator connected with an InGaAs photodetector. A polarization synthesizer was used to set the qTM-polarization of the probe light and a polarizing cube was introduced into the outcoupled light beam in order to ensure the polarization settings, thereby achieving a polarization extinction ratio of $\geq 20 \mathrm{~dB}$ during the experiments.

In case of the ring resonators, qTE-polarization selective grating couplers were used in order to couple the light into the photonic sensor chips and polarization paddlers were applied to maximize the coupled probe signal. During the measurements, the NIR laser was continuously swept over the desired wavelength range and the transmitted intensity was datalogged.

\subsection{Photonic Sensor Characterization}

The homogeneous sensing experiments were performed with $\mathrm{NaCl}$ solved in DI water in order to characterize the fabricated evanescent field refractive index sensors. First, a reference measurement with pure DI water was carried out and then liquids with different percentile mass concentrations of $\mathrm{NaCl}$ were dropped onto the resonators such that the chips were homogeneously covered with a thin film of the analyte. The same resonators were used for all measurements and were carefully cleaned with acetone, isopropanol and rinsed with DI water after each experiment. The measurement results and the sensitivities per refractive index unit are presented in Fig. 4. The graphs show that a $1 \% \mathrm{NaCl}$ concentration cause a resonance wavelength shift of about $0.8 \mathrm{~nm}$ for the $10-\mu \mathrm{m}$ disk and $84 \mathrm{pm}$ for the $10-\mu \mathrm{m}$ ring resonator, respectively. The refractive index change of $1 \% \mathrm{NaCl}$ that is diluted with $\mathrm{DI}$ water is reported to be 0.0017 per mass percent at $20^{\circ} \mathrm{C}$, which results in a refractive index change of 1.3105 to 1.32765 in the given range $(0 \%$ to

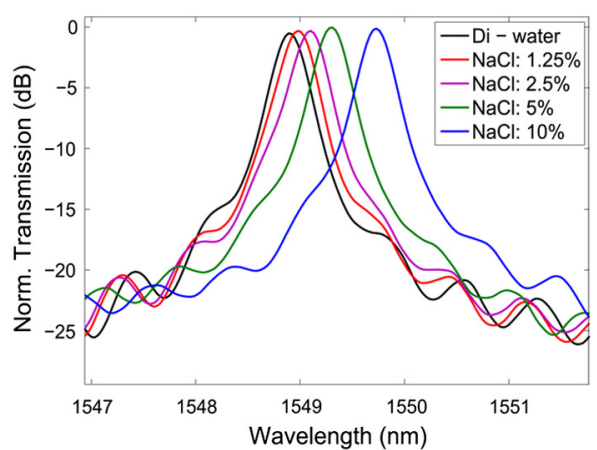

(a)

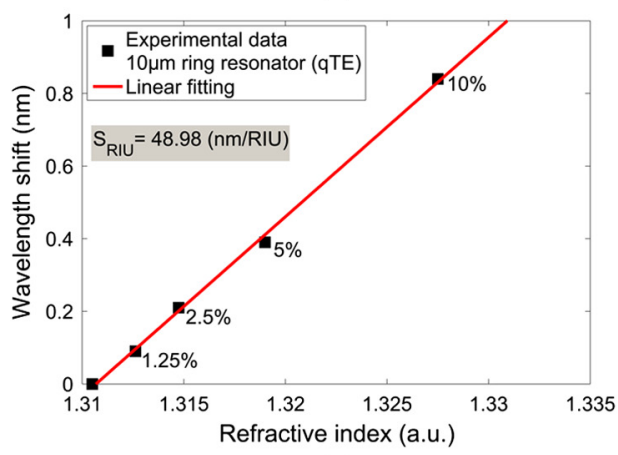

(c)

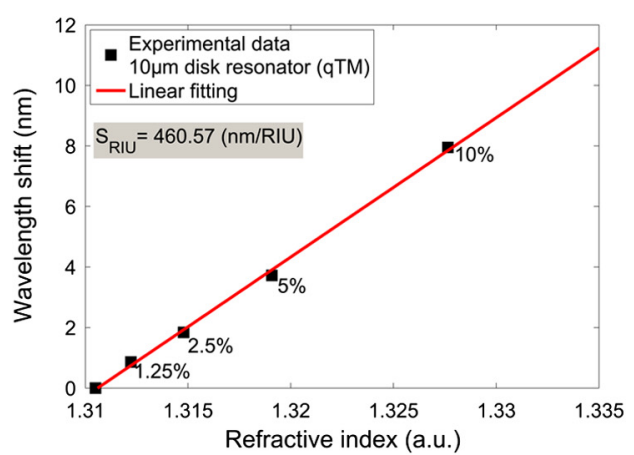

(b)

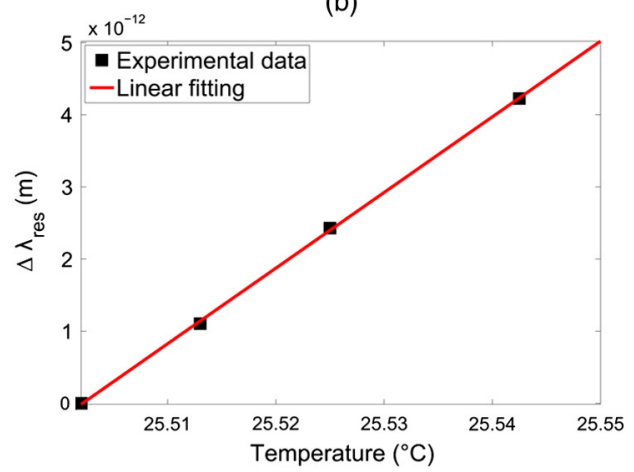

(d)

Fig. 4 Measurement results of the resonance peak wavelength shift according to $1.25 \%, 2.5 \%$, $5 \%$, and $10 \%$ of $\mathrm{NaCl}$-dispersed in deionized (DI) water: (a) typical drop port signal of a $10-\mu \mathrm{m}$ ring resonator in add/drop configuration.(b) Sensitivity of the microdisk resonator for a qTM whispering gallery mode. (c) Sensitivity of the ring resonator for qTE-mode. (d) Detection limit of the measurement setup determined by the linear thermo-optic effect induced resonance peak shift. 
Lipka et al.: Label-free photonic biosensors fabricated with low-loss hydrogenated...

10\%). ${ }^{17}$ From the measurements, the intrinsic sensitivities were determined to be $S_{\mathrm{RIU}}=460$ and $S_{\mathrm{RIU}}=50 \mathrm{~nm} / \mathrm{RIU}$. The mean $Q$-factors of the resonances that were used to determine that the sensitivities were $\approx 10^{4}$ which results in a resonator-based intrinsic LoD for homogeneous sensing of $\mathrm{LoD}=3.3 \times 10^{-4}$ for the disk and $\mathrm{LoD}=3.2 \times 10^{-3}$ for the ring resonator at $1550 \mathrm{~nm}$ wavelength, respectively. It should be noted that this intrinsic LoD is only attributed to the resonator linewidth and can be greatly improved by the readout system (typically by a factor of $10^{2}-10^{3}$ ). The wavelength sensitivity of our setup was determined via the linear thermo-optic effect by applying small temperature variations by the TEC-based chip mount that resulted in $\leq \pm 0.1 \mathrm{pm}$ resolution after Lorentz-fitting the resonance peak with a quality factor of $Q=8500$ and an $1 \mathrm{~h}$ warm-up phase of the entire equipment as presented in Fig. 4(d).

The surface sensing experiments were performed with functionalized a-Si:H ring resonators with a radius of $20 \mu \mathrm{m}$, a FSR of about $4.5 \mathrm{~nm}$, and a $Q$-factor of 7000 in aqueous solutions; more details about similarly fabricated resonator devices on the a-Si:H material platform are given elsewhere. ${ }^{18}$ First, a reference measurement is performed with a functionalized chip and the sensor surface covered with pure DI water. Then, the BSA proteins dissolved in DI water solution with a concentration of $1 \mathrm{mg} / \mathrm{ml}$ were used to homogeneously cover the whole chip. The sensor is exposed to this solution $(\approx 100 \mu \mathrm{l})$ for $1 \mathrm{~h}$ and subsequently cleaned with DI water to wash away any nonbound molecules. In order to keep the same process conditions as for the reference experiment, the chip is again covered with DI water and the spectral response is measured. The overall wavelength shift was determined to be $1.25 \mathrm{~nm}$. The results are shown in Fig. 5. From finite element method simulations, such a wavelength shift corresponds to a mean molecular layer thickness $\left(t_{L}\right)$ of $\approx 8 \mathrm{~nm}$ bound on the resonator surface. The simulations were performed with a thin molecular layer with a refractive index of $n=1.45$, and the other material properties as reported in Sec. 2. With the given molecular layer thickness $t_{\mathrm{ml}}$, the mass of this layer $M_{\mathrm{ml}}$ can be calculated according to

$$
M_{\mathrm{ml}}=t_{\mathrm{ml}} \cdot A_{\mathrm{sur}} \cdot \rho_{\mathrm{prot}},
$$

with $A_{\text {sur }}$ as waveguide surface area, and $\rho_{\text {prot }}$ as the density of dry molecules (for BSA: $\rho_{\text {prot }}=1.33 \mathrm{~g} / \mathrm{cm}^{3}$ ). From this formula, an adsorbed molecular mass on the resonator surface of $M_{\mathrm{ml}}=1.306 \mathrm{pg}$ was determined for the experiments. For a realistic wavelength accuracy of $1 \mathrm{pm}$ as minimal detectable wavelength shift resolution, molecular masses down to a femtogram or $8.5 \mathrm{pg} / \mathrm{mm}^{2}$ can be detected by the proposed a-Si:H microring resonator sensor with the given dimensions.

The experimental results of Fig. 4 clearly prove that for homogeneous sensing experiments, it is more reasonable to perform the measurements with a weakly confinent resonant mode as is the

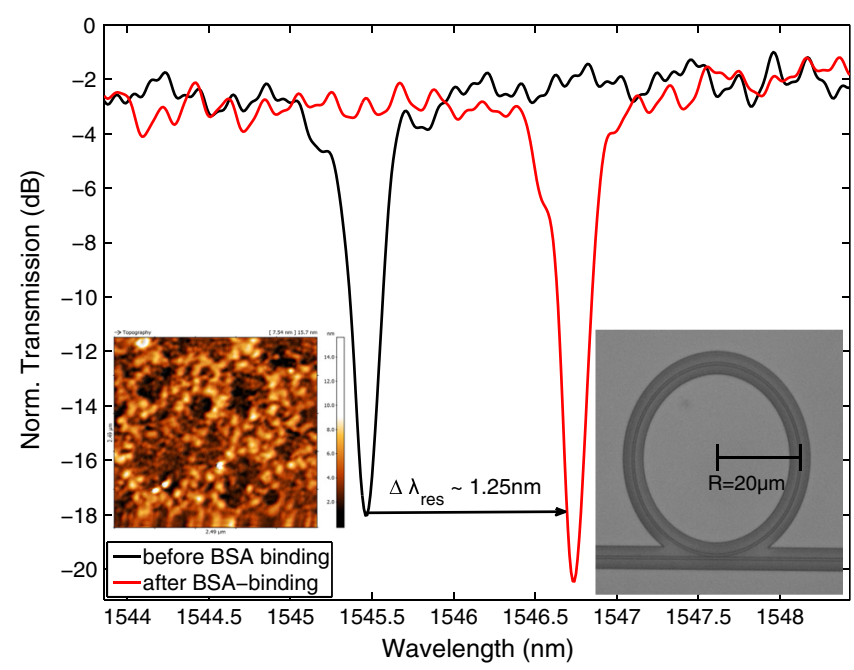

Fig. 5 Measurement results of a $20-\mu \mathrm{m}$ ring resonator covered with BSA proteins. An AFM picture of the immobilized surface and a light microscope picture of the resonator are inset. 
Lipka et al.: Label-free photonic biosensors fabricated with low-loss hydrogenated...

case for the qTM mode, which has a stronger interaction with the analyte and consequently results in a higher sensitivity; in this case, the improvement is nearly a factor of 10 . For surface sensing experiments, the main interaction of the evanscent field happens in a thin molecular layer sticked close to the sensor surface so that here a weak confinement does not necessarily improve the performance. For convenience to use the grating couplers (designed for qTE-mode) instead of cleaving the chip facets, the measurements were performed with the qTE-mode. The FEM simulations indicate that the qTM-mode would yield a 1.6 times lower detection limit. From the recent results, we suggest that a-Si:H is an attractive photonic material for lab-on-chip sensing applications, especially when combined with microfluidic channels supporting a well-defined analyte flow rate and if functionalized markers with receptors are employed which facilitate a selective detection of target molecules. The experiments show that a procedure of piranha etch, aminosilanization, and glutaraldehyde functionalization is process-compatible with lowloss amorphous silicon material, and the results from the optical characterization of a ring resonator with BSA protein adsorpted to the ring surface reveal that molecular masses down to the fg region or a surface coverage of $8.5 \mathrm{pg} / \mathrm{mm}^{2}$ can be detected for a conservative assumption of a 1-pm wavelength accuracy of the readout system. Further improvements are expected by a refinement of the waveguide cross-section, an improvement of the $Q$-factor by employing less strong absorbing buffer solutions and by an optimization of the resonator functionalization procedure.

\section{Conclusion}

Label-free photonic resonators for lab-on-chip biosensors that were fabricated with low-loss hydrogenated amorphous silicon are presented. Homogeneous sensing with $\mathrm{NaCl}$ and surface sensing experiments with immobilized bovine serum albumin proteins is demonstrated. A sensitivity as high as $460 \mathrm{~nm} / \mathrm{RIU}$ was measured for NaCl-solved in DI water for a $10-\mu \mathrm{m}$ disk resonator applying the qTM-mode, whereas about $50 \mathrm{~nm} / \mathrm{RIU}$ was determined for a $10-\mu \mathrm{m}$ ring and qTE mode. The intrinsic limits of detection were calculated to be $\operatorname{LoD}=3.3 \times 10^{-4}$ for the disk resonator and $\mathrm{LoD}=3.2 \times 10^{-3}$ for the ring resonator at $1550-\mathrm{nm}$ wavelength. The potential to measure biomolecules with high sensitivity was experimentally demonstrated by functionalized amorphous silicon ring resonators that allow a binding of BSA proteins with the capability to detect down to the clinically relevant femtogram region. Low-loss hydrogenated amorphous silicon material is a promising photonic platform to be employed in a variety of different lab-on-chip sensing areas, including chemical, medical, biosensing, and diagnostic screening applications.

\section{Acknowledgments}

The authors would like to thank the German Research Foundation (DFG grant FOR-653) for funding. Ralf Steingrüber from Fraunhofer Heinrich Hertz Institute (HHI-Berlin) is acknowledged for performing the electron-beam lithography process. T.L. performed the simulations, fabricated the devices, did the measurements and wrote the article. L.W. assisted parts of the measurements and contributed pictures. L.H. functionalized the sensors and contributed the corresponding writing part. H.K.T. and J.M. discussed and reviewed the paper.

\section{References}

1. K. De Vos et al., "Multiplexed antibody detection with an array of silicon-on-insulator microring resonators," IEEE Photonics J. 1(4), 225-235 (2009), http://dx.doi.org/10 .1109/JPHOT.2009.2035433.

2. D.-X. Xu et al., "Label-free biosensor array based on silicon-on-insulator ring resonators addressed using a wdm approach," Opt. Lett. 35(16), 2771-2773 (2010), http://dx.doi.org/ 10.1364/OL.35.002771.

3. A. Harke, M. Krause, and J. Müller, "Low-loss singlemode amorphous silicon waveguides," Electron. Lett. 41(25), 1377-1379 (2005), http://dx.doi.org/10.1049/el:20052387.

4. T. Lipka et al., "Low-loss multilayer compatible a-Si:H optical thin films for photonic applications," J. Eur. Opt. Soc. Rap. Publi. 7, 12033 (2012), http://dx.doi.org/10.2971/jeos.2012 .12033 . 
Lipka et al.: Label-free photonic biosensors fabricated with low-loss hydrogenated...

5. L. Fan et al., "Direct fabrication of silicon photonic devices on a flexible platform and its application for strain sensing," Opt. Express 20(18), 20564-20575 (2012), http://dx.doi.org/ 10.1364/OE.20.020564.

6. N. Rajabi et al., "Microfluidic device for the continuous preparation of eukaryotic cells for metabolic analysis," in 26th Int. Conf. Micro Electro Mechanical Systems (MEMS), Taipei, pp. 259-262 (2013), http://dx.doi.org/10.1109/MEMSYS.2013.6474227.

7. J. Kang et al., "Multi-stacked silicon wire waveguides and couplers toward $3 \mathrm{~d}$ optical interconnects," Proc. SPIE 8630, 863008 (2013), http://dx.doi.org/10.1117/12.2009456.

8. M. S. Luchansky and R. C. Bailey, "High-q optical sensors for chemical and biological analysis," Anal. Chem. 84(2), 793-821 (2012), http://dx.doi.org/10.1021/ac2029024.

9. F. Vollmer and L. Yang, "Review label-free detection with high-q microcavities: a review of biosensing mechanisms for integrated devices," Nanophotonics 1(34), 267-291 (2012), http://dx.doi.org/10.1515/nanoph-2012-0021.

10. V. M. Passaro et al., "Chemical sensors based on photonic structures," Chap. 5 Adv. Chem. Sens., W.Wang, Ed., ISBN: 978-953-307-792-5 (2012), http://dx.doi.org/10.5772/30719.

11. F. Dell'Olio and V. M. Passaro, "Optical sensing by optimized silicon slot waveguides," Opt. Express 15, 4977-4993 (2007), http://dx.doi.org/10.1364/OE.15.004977.

12. K. F. Palmer and D. Williams, "Optical properties of water in the near infrared," J. Opt. Soc. Am. 64, 1107-1110 (1974), http://dx.doi.org/10.1364/JOSA.64.001107.

13. L. Chrostowski et al., "Silicon photonic resonator sensors and devices," Proc. SPIE 8236, 823620 (2012), http://dx.doi.org/10.1117/12.916860.

14. T. Lipka et al., "High q-factor hydrogenated amorphous silicon microdisk resonators," in 17th Opto-Electronics and Communications Conf. (OECC), Busan, pp. 873-874 (2012), http://dx.doi.org/10.1109/OECC.2012.6276673 .

15. P. J. L. Bell and P. Karuso, "Epicocconone, a novel fluorescent compound from the fungus epicoccumnigrum," J. Am. Chem. Soc. 125, 9304-9305 (2003), http://dx.doi.org/10.1021/ ja035496+.

16. T. Lipka, J. Amthor, and J. Müller, "Process and device uniformity of low-loss a-Si:H," in IEEE Photonics Conf. (IPC), Burlingame California, pp. 923-924 (2012), http://dx.doi.org/ 10.1109/IPCon.2012.6359297.

17. H. Su and X. G. Huang, "Fresnel-reflection-based fiber sensor for on-line measurement of solute concentration in solutions," Sens. Actuators B: Chem. 126(2), 579-582 (2007), http:// dx.doi.org/10.1016/j.snb.2007.04.008.

18. T. Lipka et al., "Travelling wave resonators fabricated with low-loss hydrogenated amorphous silicon," Proc. SPIE 8767, 876707 (2013), http://dx.doi.org/10.1117/12 .2018509 .

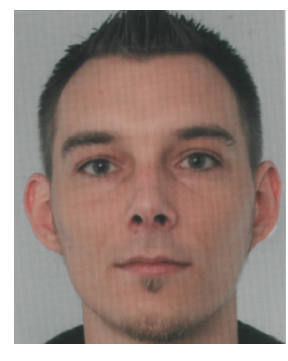

Timo Lipka received his Dipl.-Ing. degree in electrical engineering from Hamburg University of Technology (TUHH) in 2008. He is currently a research associate at the Institute of Micro System Technology working toward his $\mathrm{PhD}$ in electrical engineering. His research interests include the process development and characterization of novel optical materials, and the modeling, design, and fabrication of nanophotonic components for telecommunication and biosensing.

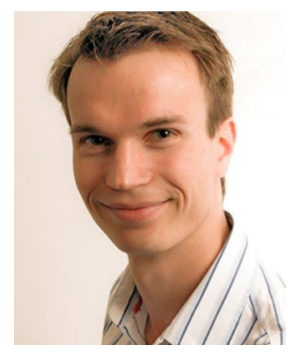

Lennart Wahn received his Dipl.-Ing. degree in electrical engineering from Hamburg University of Technology (TUHH) in 2011. He is currently a research associate at the Institute of Micro System Technology working toward his $\mathrm{PhD}$ in electrical engineering. His reasearch interests include MEMS and analog/digital electronical design. 


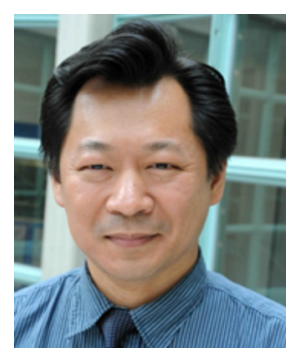

Hoc Khiem Trieu studied physics at Aachen University of Technology (RWTH) and received his $\mathrm{PhD}$ in electrical engineering from the University of Duisburg. His dissertation was about flexible silicon microstructures for integration of 3D microsystems. After 17 years in applied research at Fraunhofer Institute of Microelectronic Circuits and Systems, where he headed the department of integrated sensors and actuators with focuses on microsensors and microimplants, he became director of the Institute of Microsystems Technology at Hamburg University of Technology (TUHH) in 2011. His research is on micro-, nano-, and biointegration with applications in microsensors, medical engineering, and life sciences.

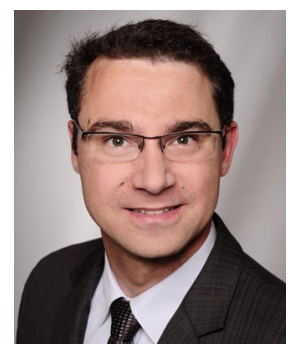

Lutz Hilterhaus has been head of the MicroBioTechnology group within the Institute of Technical Biocatalysis at Hamburg University of Technology (TUHH) since 2009. He received his $\mathrm{PhD}$ (2008) from the TUHH for research on the development of a reactor concept for the enzymatic production of novel ester oils under the supervision of Professor Andreas Liese and in close cooperation with Evonik-Goldschmidt. Afterwards he carried out his postdoctoral research in the group of Professor Uwe T. Bornscheuer at the Ernst-Moritz-Arndt University Greifswald. In the summer of 2008, he received the Karl-Heinz-Ditze Award for special achievements in engineering sciences.

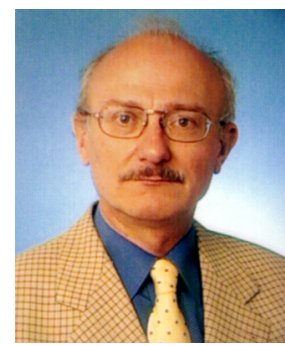

Jörg Müller studied electrical engineering at the Technical University Braunschweig, Germany. He obtained his diploma in 1969 and started his PhD studies at the Institute of HF-Technology at TU Braunschweig (H.-G. Unger), receiving his $\mathrm{PhD}$ in 1972. In 1979, he finished his Habilitation there. He worked as head of R\&D high-frequency diodes at Siemens AG, Germany, until 1983. From then to 2012 he was head of the Institute of Microsystems Technology at the Hamburg University of Technology, Germany. His research interest includes microsystems for biotechnology, gas analytics, and minimal invasive medicine, as well as integrated optics for telecommunications and sensors. In 2009, his research on a planar integrated micro mass spectrometer (PIMMS) was honored with the AMA innovation award. 